\title{
VALIDAÇÃO ANALÍTICA EM CLAE-UV PARA MONITORAÇÃO PLASMÁTICA DA METILPREDNISOLONA E DO SUCCINATO DE METILPREDNISOLONA
}

\author{
Analytical method validation of methylprednisolone and methylprednisolone sodium succinate in \\ plasma using hplc-uv technique
}

\author{
Luiz C. da Cunha ${ }^{1 * *}$, Pedro I. S. Ramalho ${ }^{1}$, Kênnia R. Rezende ${ }^{2}$, Carlos H. C. Marinho ${ }^{2}$, \\ Emílio C. de Oliveira ${ }^{2}$, Nílzio A. da Silva ${ }^{3}$ \\ ${ }^{1}$ Núcleo de Estudos e Pesquisas Tóxico-farmacológicas (NEPET-UFG). \\ ${ }^{2}$ Faculdade de Farmácia da Universidade Federal de Goiás. \\ ${ }^{3}$ Hospital das Clínicas da UFG - Ambulatório de Reumatologia.
}

Recebido em 28/09/2004 - Aceito em 23/11/2004

\begin{abstract}
RESUMO: A validação da técnica analítica para dosagem da metilprednisolona (MP) e seu éster succinato sódico (MPSS) em plasma humano de pacientes lúpicos (LES) foi desenvolvida em CLAE utilizando a triancinolona [500 $\mu \mathrm{L} / \mathrm{mL}$ ] como padrão interno. A faixa dinâmica compreendida pela MP e MPSS foi de 10,0 a 1,0 $\mu \mathrm{g} / \mathrm{mL}$ adicionados em plasma As condições cromatográficas empregadas foram sistema isocrático com fase móvel acetonitrila:água:ácido acético (47:50:03) sob fluxo de 0,8 $\mathrm{mL} / \mathrm{min}$, coluna $\mathrm{RP}_{18}(25 \mathrm{~cm} \mathrm{e} 0,4 \mathrm{~mm}$ d.i.) e detector UV $(\lambda=254 \mathrm{~nm})$. A validação analítica apresenta-se conforme normas internacionais e legislação sanitária vigente no Brasil. Além de excelente linearidade $\left(R_{M P}=0,99933 ; R_{M P S s}=0,99638\right)$, a técnica demonstrou elevada precisão intra- e interdia (<7\%), com adequada exatidão (>90\%) e recuperação (> $85 \%)$. O limite de quantificação foi de $0,25 \mathrm{~g} / \mathrm{mL}$. Adicionalmente, a técnica demonstrou ser robusta, estável e seletiva podendo ser empregada na quantificação plasmática da metilprednisolona em pacientes sob pulsoterapia e, também, em situações de intoxicação aguda e controle terapêutico de medicamentos.
\end{abstract}

PALAVRAS-CHAVE: Validação; Técnica Analítica; HPLC; Metilprednisolona; Lúpus Eritematoso Sistêmico.

ABSTRACT: The HPLC-UV analytical method validation aimed the quantification of methylprednisolone (MP) and its succinate sodic ester (MPSS) in human plasma of patients with lupus. Triancinolone $(500 \mathrm{~g} / \mathrm{mL})$ was used as internal standard the standards MP and MPSS were spiked in plasma in the range of 10 and $1.0 \mathrm{~g} / \mathrm{mL}$. The chromatographic conditions employed an isocratic mobile phase (acetonitrile:water:acetic acid 47:50:03), flow rate of $0.8 \mathrm{~mL} / \mathrm{min}$ and a RP18 column $(25 \mathrm{~cm}$ and $0.4 \mathrm{~mm}$ i.d.) coupled to an UV detector set at $254 \mathrm{~nm}$. The parameters of analytical validation are in compliance with international regulations and with the Brazilian sanitary legislation. Beyond excellent linearity $\left(R_{M P}=0.99933 ; R_{M P S S}=0.99638\right)$, it showed high intraday and interday precision $(<7 \%)$, good accuracy $(94,0 \pm 2.3 \%)$, adequate limit of quantification $(0.25 \mathrm{~g} / \mathrm{mL})$ and good recovery $(>$ $85 \%$ ). Additionally, the technique have shown to be robust, steady, selective and can be used in the dosage of methylprednisolone in patients under pulsotherapy and also in situations of acute poisoning and therapeutical monitoring.

KEYWORDS: Validation; Analytical Technique; HPLC-UV; Methylprednisolone; Lupus. 


\section{INTRODUÇÃO}

A metilprednisolona (MP) é um glicocorticóide sintético usado por via oral ou parenteral como antiinflamatório ou agente de imunomodulação. Dentre suas indicações está o tratamento de pacientes com lupus eritematoso sistêmico (LES) através de esquema de altas doses (1000 mg de succinato sódico de metilprednisolona - MPSS por infusão rápida em 1 hora) denominado pulsoterapia.

A MP ocorre como pó cristalino branco à praticamente branco, inodoro e cristalino. Apresenta ponto de fusão = $228-237^{\circ} \mathrm{C},[]_{\mathrm{D}}^{20}=+83^{\circ}\left(\right.$ em dioxano), $\mathrm{UV}_{\mathrm{MAX}}($ etanol $95 \%)=243 \mathrm{~nm}(\mathrm{M}=14875)$. É parcialmente solúvel em álcool, em dioxano, e em metanol; ligeiramente solúvel em acetona e em clorofórmio, e pouco solúvel em éter. É praticamente insolúvel em água. O sal MPSS é muito solúvel em água, etanol e metanol (BUDAVARI, 1989).

Há poucas referências a respeito da cinética da MP utilizada sob altas doses em pacientes de LES (BERTONI, 1994; ICHIKAWA, 1994; RÊGO, 1994) e de técnicas de quantificação da MP em HPLC com doses do fármaco iguais ou superiores a $1000 \mathrm{mg}$ (LAWSON, 1985; SHAH et al., 1987; VREE et al., 1999). Dessas, somente a de LAWSON (1985) utiliza amostra de pacientes de LES sob pulsoterapia para desenvolver técnica analítica em HPLC, porém sem quantificar o succinato de metilprednisolona.

Objetivando estudar o perfil cinético da MP em regime de pulsoterapia, foi desenvolvida e validada no Laboratório de Análises Toxicológicas da Faculdade de Farmácia da UFG técnica analítica para quantificação plasmática deste fármaco e seu éster succinato sódico, utilizando como método a cromatografia líquida de alta eficiência sob detecção ultravioleta (HPLC-UV) (RAMALHO, 2002).

\section{MATERIAL E MÉTODOS}

\section{Reagentes}

Os padrões secundários de mp (11,17,21-triidroxi-6-metil-1,4-pregnadieno-3,20-diona) e triancionolona (padrão interno) foram obtidos da sigma-aldrich ${ }^{\text {tm }}$ company e pharmacia artesanal ${ }^{\circledR}$, respectivamente, ambos com laudo de qualidade. os solventes orgânicos utilizados foram adquiridos da baker ${ }^{\circledR}$, todos com grau de pureza para cromatografia líquida de alta eficiência. utilizou-se água ultrapura (waters/millipore ${ }^{\circledR}$ ) na preparação de soluçõestampão e regeneração do cromatógrafo líquido.

\section{Condições cromatográficas}

Na determinação quantitativa da MP e do MPSS no plasma empregou-se cromatógrafo Varian ${ }^{\circledR}$ (ProStar 340 ), equipado com injetor Rheodyne ${ }^{\circledR}$, pré-coluna e coluna $\mathrm{RP}_{18}(25 \mathrm{~cm}$ e $0,4 \mathrm{~mm}$ d.i.) e fluxo de $0,6 \mathrm{~mL} / \mathrm{min}$, com detecção ultravioleta (UV) em $\lambda=254 \mathrm{~nm}$, monitorados em registrador e integrador Varian ${ }^{\circledR}$ acoplados a microcomputador com software para registro, integração, gerenciamento e tratamento dos dados analíticos.

A fase móvel, acetonitrila:água:ácido acético (47:50:3 v/v) foi desgaseificada com o auxílio de banho de ultrassom e bomba de pressão reduzida. as análises diárias através de hplc envolveram: preparação de curva de calibração (padrões em triplicata), controles de qualidade internos (triplicata), branco de plasma (duplicata) e amostras dos pacientes (triplicata).

\section{Preparação das amostras}

Após administração de $1000 \mathrm{mg}$ do fármaco por infusão rápida em solução fisiológica $(1 \mathrm{~h})$, colheram-se amostras de sangue seriadas através de punção venosa, nos tempos zero, 0,$5 ; 0,75 ; 1,0 ; 1,5 ; 2,0 ; 3,0 ; 4,0 ; 6,0$; 8,$0 ; 10,0$ e 24,0 h, sendo que a veia foi mantida através de cateter Gelco ${ }^{\circledR}$ heparinizado. O protocolo deste estudo foi aprovado pelo Comitê de Ética e Pesquisa da UFG.

O sangue foi transferido para tubo plástico contendo o anticoagulante sal sódico do ácido etileno-diaminotetracético (EDTA; 1 gota/5 mL sangue), centrifugado a $4.000 \mathrm{rpm}$ (Centrífuga FANEM Excelsa Baby Mod. 240) por 10 minutos sendo então o plasma armazenado a $-20^{\circ} \mathrm{C}$ até análise por HPLC. 
Durante a validação analítica foram utilizadas bolsas de plasma cedidas pelo Banco de Sangue do Hospital das Clínicas da UFG. Amostras de plasma foram preparadas (branco e concentrações variadas de MP e MPSS a 10, $5,0,2,5,1,0,0,5$ e $0,1 \mu \mathrm{g} / \mathrm{mL}$ ) e aliquotadas em tubos de ensaio criogênicos, sendo a seguir armazenadas em freezer a $-20{ }^{\circ} \mathrm{C}$. Posteriormente, adiciona-se $10 \mu \mathrm{L}$ do padrão interno (triancinolona - TR, $500 \mathrm{~g} / \mathrm{mL}$ em metanol) a $500 \mu \mathrm{L}$ do plasma, procedendo a extração do analito. Adiciona-se $3,0 \mathrm{~mL}$ de acetato de etila, levandose ao vórtex por $1 \mathrm{~min}$ e centrifugação a $4.000 \mathrm{rpm} / 10 \mathrm{~min}$. Descarta-se a fase aquosa. A fase orgânica é imersa em nitrogênio líquido (N2) para congelamento de resíduo de água. O extrato orgânico é transferido para outro tubo de ensaio e, em seguida, levado ao evaporador de solventes a $50^{\circ} \mathrm{C}$ sob fluxo de nitrogênio até a secagem. O resíduo é dissolvido em $200 \mu \mathrm{L}$ de fase móvel (FM), sendo injetados $20 \mu \mathrm{L}$ de amostra em HPLC. Todos os passos da técnica analítica desenvolvida estão ilustrados na Figura 1.

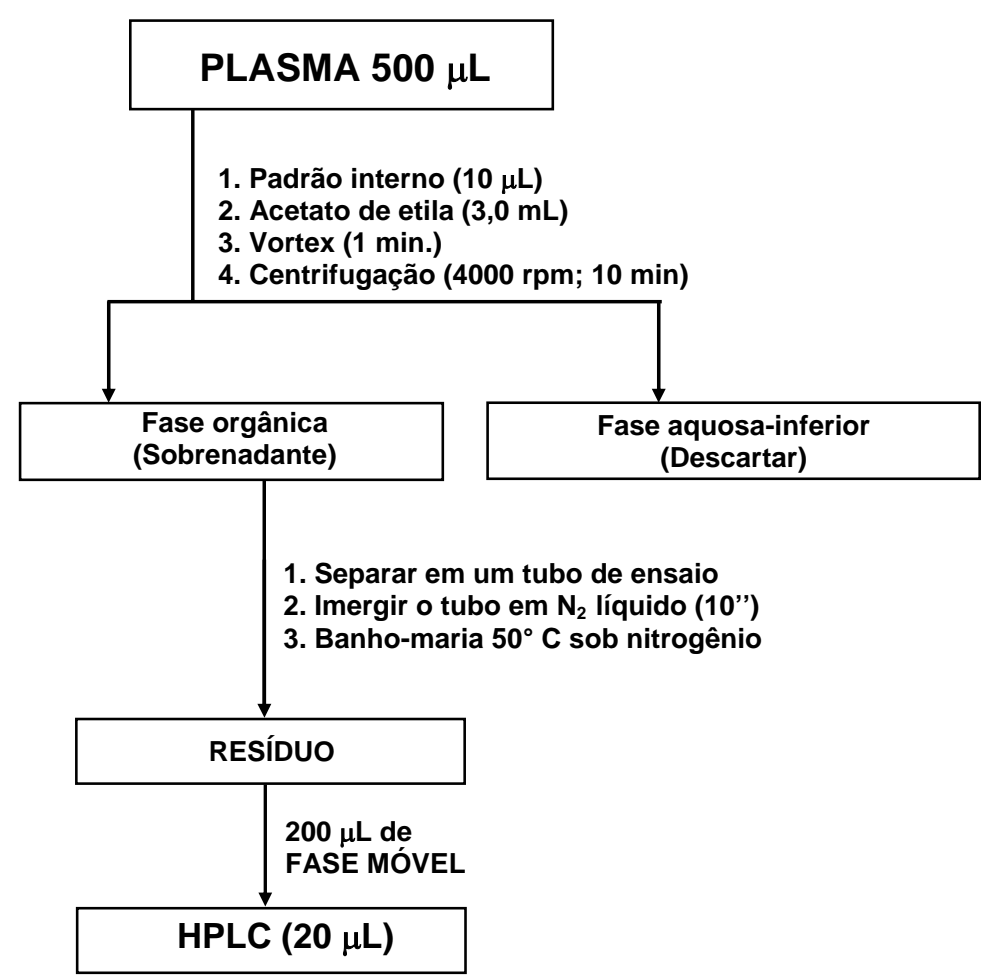

Figura 1. Etapas do procedimento analítico para MPSS e MP.

\section{Validação da técnica analítica}

As concentrações plasmáticas das amostras foram calculadas através de fatores $(F)$ relativos à razão de área $(R A)$ do padrão de MP (MP) contra a RA do padrão interno (PI), como equações a seguir: $R A_{M P}=A_{A M O S T R A} / A_{P I} e$ $F_{M P}=C_{P A D R A ̃ O} / R A_{M P}$, onde: $R A$ = razão de área; $M P=M P ; P I=$ padrão interno.; $A_{P i}=$ área do padrão interno; $F=$ fator e $C_{\text {PADRÃO }}=$ concentração do padrão MP. As mesmas equações foram aplicadas para a MPSS.

Os fatores obtidos através da integração dos picos dos padrões alto, médio e baixo em triplicata e com CV $\leq$ $10 \%$ foram multiplicados pelas respectivas razões de área das amostras, obtendo-se desta forma as concentrações de MP.

A validação da técnica analítica foi efetuada após otimização do procedimento e adaptações às condições do laboratório com determinação dos parâmetros limite de quantificação (LOQ), limite de deteç̧ão (LOD), recuperação relativa, recuperação absoluta, linearidade, precisão, sensibilidade, seletividade, exatidão, abrangência, estabilidade dos analitos e robustez da técnica empregada. Construíram-se curvas de calibração a partir de solução-padrão estoque de $1 \mathrm{mg} / \mathrm{mL}$, em plasma humano livre do fármaco (branco de plasma), de forma a se obter concentrações na faixa compreendida entre 0,05 e $10 \mathrm{~g} / \mathrm{mL}$, além de concentrações $1 \mathrm{~g} / \mathrm{mL}, 5$ $\mathrm{g} / \mathrm{mL}$ e $10 \mathrm{~g} / \mathrm{mL}$ para os padrões baixo, médio e alto, respectivamente (PENG, 1990; QUATTROCCHI, 1992; BRESSOLLE, 1996; CUNHA, 1998; BRASIL, 1999; BRASIL, 2002). 


\section{RESULTADOS}

Cromatogramas representativos são apresentados nas figuras 2, 3 e 4 . Curvas de calibração para a metilprednisolona e o succinato de metilprednisolona são apresentadas na figura 5. Já nas Tabelas I e II são apresentados os resultados da validação analítica, incluindo os parâmetros exatidão, precisão intradia e interdia, seletividade, abrangência, robustez, estabilidade dos analitos, recuperação, resolução, LOD e LOQ. Apresentamse, nos quadros 1 e 2 parâmetros de técnicas analíticas desenvolvidas em HPLC para a quantificação da metilprednisolona, revisados da literatura. Finalmente, na figura 6, são apresentados o perfil cinético de concentração vs tempo da MP e da MPSS de uma paciente com LES sob pulsoterapia com succinato de metilprednisolona $1000 \mathrm{mg}$ i.v., $1 \mathrm{~h}$ infusão, bem como os parâmetros cinéticos representativos dos dois fármacos [concentração máxima, $C_{M A X}(\mathrm{~g} / \mathrm{mL})$; tempo para atingir a concentração máxima, $T_{M A X}(h)$; constante de eliminação, $K_{e l}\left(h^{-1}\right)$; meia-vida de eliminação, $T_{(1 / 2)}(h)$; área sob a curva de concentração vs tempo, $A U C_{T}$ ( $\mathrm{g} / \mathrm{mL}$.h); clearance total, $C l_{T}(\mathrm{~mL} / \mathrm{min})$ e volume de distribuição, $V d(\mathrm{~L} / \mathrm{Kg})$ ], calculados por meio do software Kinetica2000 ${ }^{\circledR}$ (InnaPhase Co.).

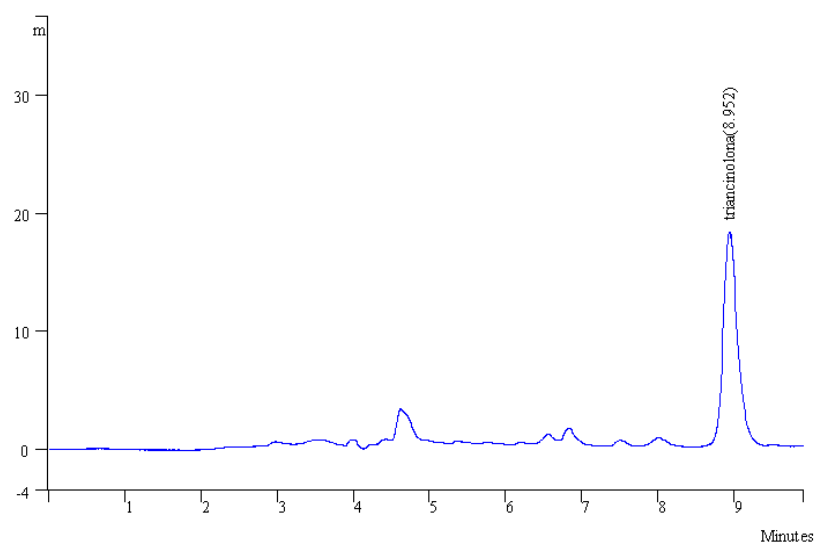

Figura 2. Cromatograma de amostra de plasma branco contendo $10 \mathrm{~g} / \mathrm{mL}$ do padrão interno triancinolona $10 \mathrm{~g} / \mathrm{mL}$.

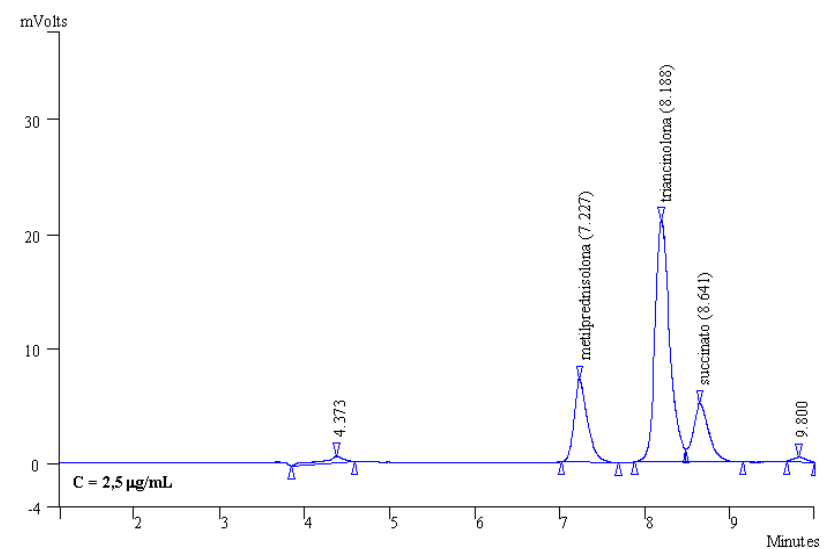

Figura 3. Cromatograma dos corticosteróides MP e MPSS em HPLC-UV a $2,5 \mathrm{~g} / \mathrm{mL}$ contendo 10 g/mL do padrão interno triancinolona. 


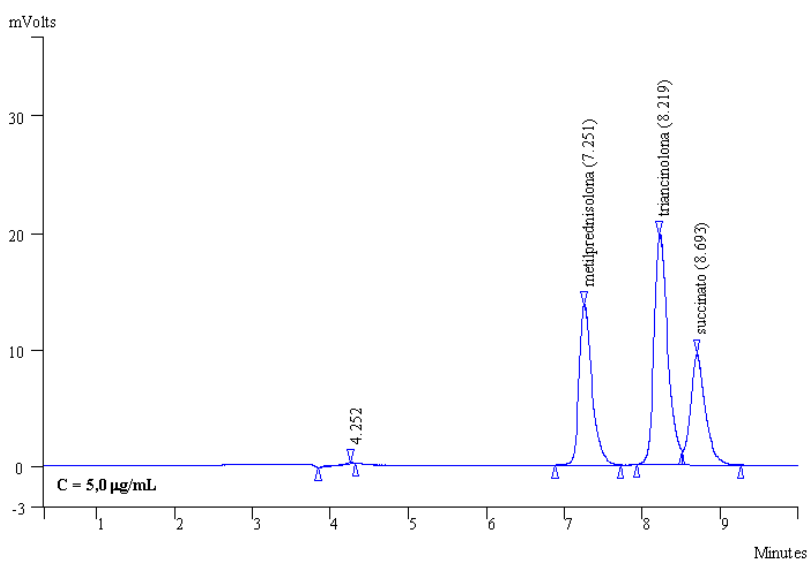

Figura 4. Cromatograma dos corticosteróides MP e MPSS em HPLC-UV a 5,0 g/mL contendo $10 \mathrm{~g} / \mathrm{mL}$ do padrão interno triancinolona.
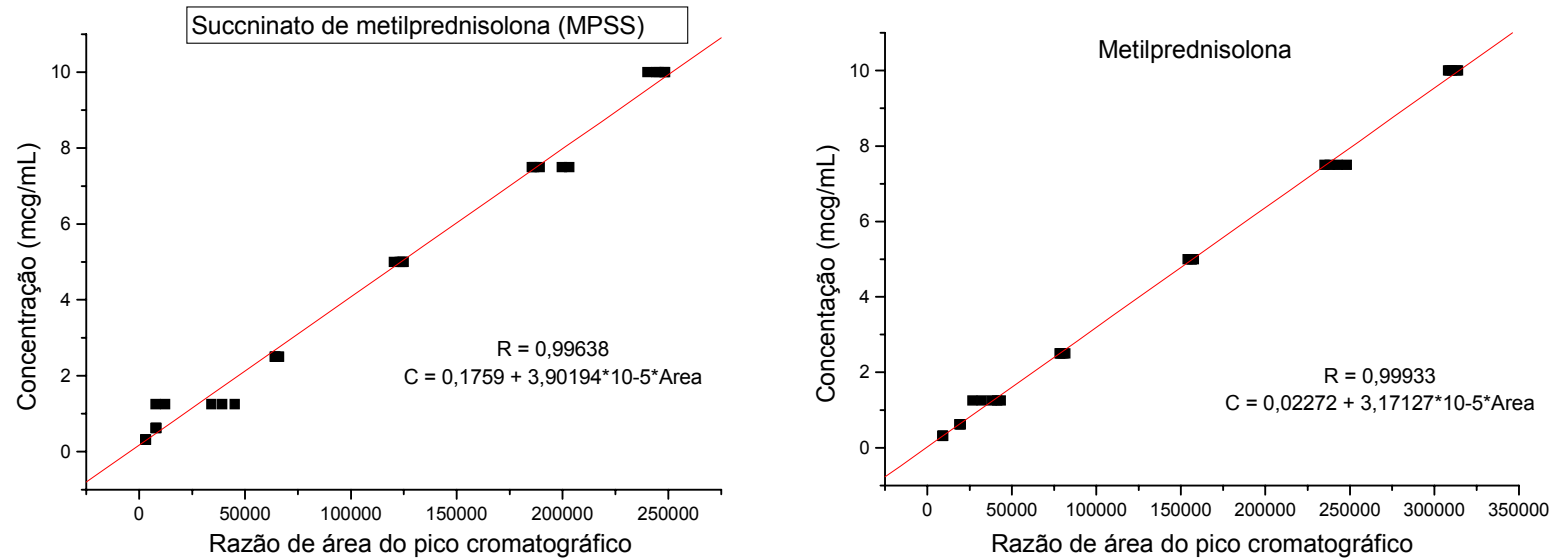

Fig 5. Curva de calibração para a análise quantitativa do succinato de metilprednisolona (esquerda) $(R=0,99638 ; E q$. Reta: $C=0,1759+$ $\left.3,90194^{*} 10^{-5}\right)$ e da metilprednisolona (direita) $\left(R=0,99933\right.$; Eq. Reta: $\left.C=0,02272+3,17127^{*} 10^{-5}\right)$ e em HPLC sob UV $=254 \mathrm{~nm}$.

Tabela 1. Cálculo da precisão intradia e interdia e exatidão para a quantificação plasmática por HPLC da metilprednisolona e do succinato de metilprednisolona.

\begin{tabular}{|c|c|c|c|c|c|c|}
\hline PADRÃO & MP & MP & MP & MPSS & MPSS & MPSS \\
\hline \multirow[t]{4}{*}{ Precisão intradia } & $\begin{array}{c}9.4 \\
10,2\end{array}$ & $\begin{array}{c}5 \\
4,8\end{array}$ & $\begin{array}{c}0.87 \\
0,9\end{array}$ & $\begin{array}{c}9.4 \\
10,1\end{array}$ & $\begin{array}{l}4.85 \\
4,75\end{array}$ & $\begin{array}{l}1.06 \\
0,92\end{array}$ \\
\hline & 9,6 & 4,75 & 0,97 & 9,65 & 4,55 & 0,93 \\
\hline & 9,75 & 4,9 & 0,84 & 9,95 & 4,65 & 0,92 \\
\hline & 9,95 & 4,4 & 0,88 & 9,3 & 4,23 & 0,94 \\
\hline Média & 9,78 & 4,77 & 0,89 & 9,68 & 4,61 & 0,90 \\
\hline DP & 0,31 & 0,23 & 0,05 & 0,34 & 0,24 & 0,06 \\
\hline CV\% & 3,16 & 4,78 & 5,46 & 3,55 & 5,17 & 6,65 \\
\hline \multirow{2}{*}{$\begin{array}{l}\text { Precisão interdia } \\
1^{\circ} \mathrm{dia}\end{array}$} & $\begin{array}{c}9.4 \\
10,1\end{array}$ & $\begin{array}{l}4.9 \\
4,5\end{array}$ & $\begin{array}{c}0.9 \\
0,87\end{array}$ & $\begin{array}{l}9.5 \\
10\end{array}$ & $\begin{array}{c}4.85 \\
4,7\end{array}$ & $\begin{array}{l}1.05 \\
0,91\end{array}$ \\
\hline & 9,55 & 4,7 & 0,96 & 9,7 & 4,5 & 0,94 \\
\hline \multirow{3}{*}{$\begin{array}{l}\text { Precisão interdia } \\
2^{\circ} \mathrm{dia}\end{array}$} & 9 & 4,9 & 0,98 & 9 & 4,9 & 1,02 \\
\hline & 9,8 & 4,6 & 0,85 & 9,9 & 4,6 & 0,9 \\
\hline & 9,9 & 4,2 & 0,87 & 9,2 & 4,2 & 0,93 \\
\hline Média & 9,63 & 4,63 & 0,91 & 9,55 & 4,63 & 0,96 \\
\hline DP & 0,39 & 0,27 & 0,05 & 0,39 & 0,26 & 0,06 \\
\hline CV\% & 4,10 & 5,74 & 5,88 & 4,12 & 5,54 & 6,45 \\
\hline \multirow[t]{2}{*}{ Recuperação (\%) } & $\begin{array}{c}9.2 \\
10,1\end{array}$ & $\begin{array}{l}4.9 \\
4,5\end{array}$ & $\begin{array}{c}0.9 \\
0,87\end{array}$ & $\begin{array}{c}9.5 \\
10\end{array}$ & $\begin{array}{c}4.85 \\
4,7\end{array}$ & $\begin{array}{l}0.89 \\
0,92\end{array}$ \\
\hline & 9,7 & 4,7 & 0,96 & 9,7 & 4,5 & 0,94 \\
\hline Exatidão & 96,7 & 94 & 91 & 97,3 & 93,7 & 91,7 \\
\hline
\end{tabular}




\section{Seção 1.01}

Tabela 2. Outros parâmetros de validação analítica dos corticosteróides metilprednisolona e succinato de metilprednisolona.

\section{Seção 1.02 PARÂMETRO DE VALIDAÇÃO}

Seção 1.04 LINEARIDADE
Seção 1.05 SELETIVIDADE
Seção 1.06 ABRANGÊNCIA
Seção 1.07 ROBUSTEZ
Seção 1.08 ESTABILIDADE
DOS ANALITOS

Seção 1.09 RECUPERAÇÃO

Seção 1.10 RESOLUÇÃo

Seção 1.11 LIMITE DE DETECÇÃO (LOD)

Seção 1.12 LIMITE DE QUANTIFICAÇÃO (LOQ)

Seção 1.13 SENSIBILIDADE

\section{Seção 1.03 RESULTADO}

- $\quad$ succinato de metilprednisolona $R=0,99638 ;$ Eq. Reta: $C=0,1759$ + $\left.3,90194 * 10^{-5}\right)$;

- metilprednisolona ( $R=0,99933$; Eq. Reta: $C=0,02272+3,17127^{*} 10^{-5}$ )

- Amostras de plasma hemolisadas e liPÊMICAS NÃO INTERFERIRAM NA tÉCNICA ANALÍTICA.

- Os fármacos seguintes não interferem no sistema cromatográfico empregado: ácido acetil salicílico, dipirona, paracetamol, hidroclortiazida, nifedipina, azatioprina, cafeína, penicilina, ampicilina, amoxicilina, cefalotina, cefalexina, metotrexato, gentamicina, tobramicina, dexametasona, betametasona, hidrocortisona e cortisol.

- É POSSível anAlisar, simultaneamente: PREDNisolona, PREDNisona, TRIANCINOLONA, METILPREDNISOLONA, SUCCINATO DE METILPREDNISOLONA, DEXAMETASONA, BETAMETASONA, HIDROCORTISONA E CORTISOL.

- MODIFICAÇÕES EM MARCAS DE SOLVENTES E OPERADOR DO EQUIPAMENTO CROMATOGRÁFICO NÃO INTERFERIRAM NA VALIDAÇÃO DA TÉCNICA.

- A (IM)PRECISÃo PARA OS PADRÕES ALto (10 G/ML), MÉdIO (5 G/ML) E BAIXo (1 G/ML) DOS ANALITOS METILPREDNISOLONA E SUCCINATO DE METILPREDNISOLONA MANTEVE-SE EM VALORES ABAIXO DE 7\%, APÓS TRÊS CICLOS DE CONGELAMENTO/DESCONGELAMENTO EM TRÊS DIAS SUCESSIVOS.

- A RECuperaÇÃo ABSOluta para os PADRÕEs ALto (10 G/ML), MÉdio (5 G/ML) E BAIXO (1 G/ML) DOS ANALITOS METILPREDNISOLONA E SUCCINATO DE METILPREDNISOLONA FOI SEMPRE MAIOR QUE $80 \%$, A SABER: MP (88 $\pm 6 \%$ ), MPSS $(85 \pm 5 \%)$ E TRIANCINOLONA (90 $\pm 7 \%)$.

- OS PICOS DE TR, MP E MPSS APRESENTARAM RESOLUÇÃO PRÓXIMA DE 1,5.

- 0,1 G/ML PARA MP E MPSS.

- $\quad 0,25$ G/ML PARA MP E MPSS.

- $\quad 0,25$ G/ML PARA MP E MPSS.

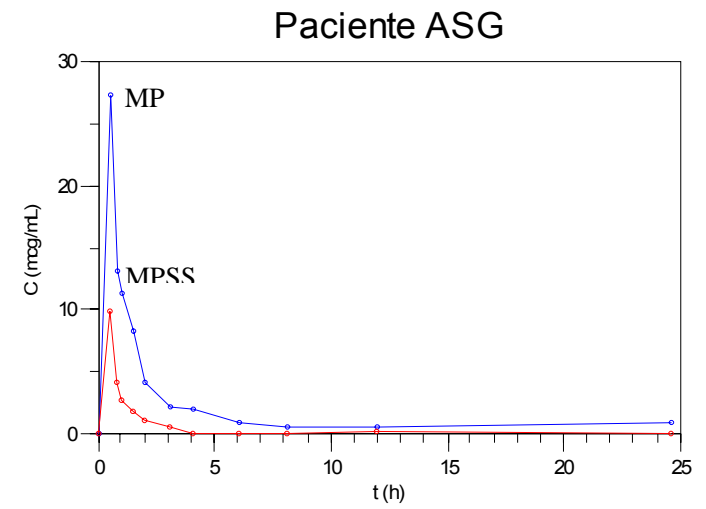

\begin{tabular}{lcc}
\hline \multicolumn{1}{c}{ Parâmetros } & MP & MPSS \\
\hline$C_{M A X}(\mathrm{~g} / \mathrm{mL})$ & 27,3683 & 9,9692 \\
$T_{M A X}(\mathrm{~h})$ & 0,57 & 0,57 \\
$K_{\mathrm{el}}\left(\mathrm{h}^{-1}\right)$ & 0,2056 & 0,4041 \\
$T_{(1 / 2)}(\mathrm{h})$ & 3,3714 & 1,7152 \\
$A U C_{T}(\mathrm{~g} / \mathrm{mL} . \mathrm{h})$ & 37,0576 & 8,67801 \\
$C_{T}(\mathrm{~mL} / \mathrm{min})$ & 449,75 & 2546,67 \\
$V d(\mathrm{~L} / \mathrm{Kg})$ & 2,73 & 7,88 \\
\hline
\end{tabular}

Figura 6. Perfil cinético e parâmetros cinéticos da MP e da MPSS em paciente submetida a tratamento de LES com succinato de metilprednisolona (1000 mg, i.v.; $1 \mathrm{~h}$ infusão). 
Quadro 1. Parâmetros de técnicas analíticas em HPLC para quantificação plasmática da MP e do MPSS colhidos em literatura especializada internacional.

\begin{tabular}{|c|c|c|c|c|c|c|c|c|c|c|c|c|c|c|}
\hline Referência & $\begin{array}{l}\text { Dose/Via } \\
\text { Adm. }\end{array}$ & $\begin{array}{l}\text { Material } \\
\text { Biol. }\end{array}$ & Analito & $\begin{array}{l}T R \\
(M P) \\
(\min )\end{array}$ & Extração & Coluna & $\begin{array}{l}\text { Sist. } \\
\text { Cromat }\end{array}$ & $(\mathrm{nm})$ & Fase móvel & $\begin{array}{l}\text { Fluxo } \\
\text { (mL/min) }\end{array}$ & $\begin{array}{c}\text { Volume } \\
\text { da } \\
\text { amostra }\end{array}$ & $\begin{array}{l}\angle O D \\
M P\end{array}$ & Padrão interno & $\begin{array}{c}N^{\circ} \text { de } \\
\text { pacientes }\end{array}$ \\
\hline $\begin{array}{l}\text { AL-HABET \& } \\
\text { ROGERS, } \\
1989\end{array}$ & $\begin{array}{l}20,40,80 \\
\text { MPSS }\end{array}$ & $\begin{array}{l}\text { Plasma, } \\
\text { saliva e } \\
\text { urina }\end{array}$ & $\begin{array}{l}\text { MP, PL e } \\
\text { MPSS }\end{array}$ & ------ & Acetato de etila & $\begin{array}{l}0,45 \times 20 \\
\mathrm{~cm} \mathrm{e} \\
\text { Zorbax }^{\odot} \\
\text { SIL-5 } 5 \mathrm{~m}\end{array}$ & Isocrático & 254 & $\begin{array}{l}\text { Diclorometano:metanol:ác. } \\
\text { acético }(95: 1: 3,75)\end{array}$ & 2,5 & $\begin{array}{l}0,5-1,0 \\
\mathrm{~mL}\end{array}$ & ------- & Triancinolona & $\begin{array}{l}9(7 \mathrm{M} \\
\text { e } 2 \mathrm{~F})\end{array}$ \\
\hline $\begin{array}{l}\text { HOPKINS et } \\
\text { al., } 1992\end{array}$ & ------- & Plasma & $\begin{array}{l}\text { MP e } \\
\text { Acetato de } \\
\text { MP }\end{array}$ & ------- & Diclorometano & $\begin{array}{c}\text { ODS } \\
5 \mu \mathrm{m} \\
250 \mathrm{x} \\
4,6 \mathrm{~mm} \text { e } \\
\mathrm{C}_{18} \\
\end{array}$ & Isocrático & 254 & $\begin{array}{c}\text { Acetonitrila:água: } \\
\text { ác.acético glacial (33:62:5) }\end{array}$ & 1,0 & $2 \mathrm{~mL}$ & $\begin{array}{c}2 \\
\mathrm{ng} / \mathrm{mL}\end{array}$ & Fluorometalona & ------- \\
\hline $\begin{array}{l}\text { KONG et al., } \\
1988\end{array}$ & $\begin{array}{l}30 \mathrm{mg} \text { de } \\
\text { MPSS } \\
\text { (i.v. bolus) }\end{array}$ & Plasma & $\underset{\mathrm{C}}{\mathrm{MPHS}, \mathrm{MPe}}$ & ------- & $\begin{array}{l}\text { Hexano:acetato } \\
\text { de etila }(1: 1) \text { e } \\
\text { ác. nítrico }\end{array}$ & $\begin{array}{c}80 \times 4 \\
\mathrm{~mm} \\
12,5 \times 4 \\
\mathrm{~mm} \\
70 \times 6 \\
\mathrm{~mm} \text { (pré) }\end{array}$ & Isocrático & 254 & $\begin{array}{c}\text { Diclorometano:hexano: } \\
\text { etanol:ác.acético glacial } \\
(69: 26: 2,3: 1)\end{array}$ & 2,0 & $1,0 \mathrm{~mL}$ & ------- & Prednisona & 1 \\
\hline $\begin{array}{l}\text { LAWSON, } \\
1985\end{array}$ & $\begin{array}{l}0,5-2 \mathrm{~g} \mathrm{de} \\
\text { MPSS (IR) }\end{array}$ & $\begin{array}{c}\text { Plasma e } \\
\text { urina }\end{array}$ & MP e MPHS & 5 & $\begin{array}{c}\text { Extrelut }^{\oplus} \mathrm{e} \\
\text { acetato de etila }\end{array}$ & $\begin{array}{l}\mathrm{C}_{18} 100 \mathrm{x} \\
5 \mathrm{~mm} \mathrm{DI}\end{array}$ & Isocrático & 251 & $\begin{array}{c}\text { acetonitrila e tampão acetato } \\
(32,5: 67,5)\end{array}$ & 1,5 & $500 \quad \mathrm{~L}$ & $\begin{array}{c}267 \\
\mathrm{nmol} / \mathrm{L}\end{array}$ & $\begin{array}{l}\text { 11-deoxi-17- } \\
\text { hidroxi-CT }\end{array}$ & 28 \\
\hline $\begin{array}{l}\text { McWHINNEY } \\
\text { et al., } 1996\end{array}$ & ------ & $\begin{array}{l}\text { Plasma e } \\
\text { urina }\end{array}$ & $\begin{array}{c}\text { C, OS, } \\
11- \\
\text { deoxicortisol, } \\
\text { MP e DX }\end{array}$ & 32 & ------ & $\mathrm{C}_{18}$ & Isocrático & 254 & $\begin{array}{l}\text { Metanol: } \\
\text { tetrahidrofurano: } \\
\text { água (3:25:72) }\end{array}$ & 1,0 & ------ & ------- & Fludrocortisona & ------ \\
\hline $\begin{array}{l}\text { MEHVAR et } \\
\text { al., 2000a }\end{array}$ & $\begin{array}{l}5 \mathrm{mg} / \mathrm{Kg} \\
\text { (MP eq.) }\end{array}$ & $\begin{array}{l}\text { Plasma } \\
\text { de rato }\end{array}$ & $\begin{array}{l}\text { MP, MD, CT, } \\
\text { MPSS }\end{array}$ & 13 & Diclorometano & $\begin{array}{c}\text { PRP-1 } \\
(5 \mu \mathrm{m}) \\
\mathrm{C}_{18}\end{array}$ & Isocrático & 250 & $\begin{array}{c}\text { acetonitrila e } 0,1 \mathrm{M} \mathrm{de} \\
\text { tampão acetato pH 5,7 } \\
(23: 77)\end{array}$ & 1,5 & $\begin{array}{c}100-500 \\
L\end{array}$ & $\begin{array}{c}0,1 \\
\mu \mathrm{g} / \mathrm{mL}\end{array}$ & $\begin{array}{l}\text { Triancinolona } \\
\text { acetonide }\end{array}$ & 4 ratos \\
\hline $\begin{array}{l}\text { MEHVAR, } \\
1999\end{array}$ & ------ & ------ & $\begin{array}{c}\text { MP e } \\
\text { MPSS e } \\
\text { DEX-MPS }\end{array}$ & $\begin{array}{c}5 \text { (MP) } \\
\text { e } \\
8,5 \\
\text { MPSS }\end{array}$ & ------ & $\begin{array}{l}\text { Coluna } \\
\text { em gel }\end{array}$ & Isocrático & 250 & $\begin{array}{c}\mathrm{KH}_{2} \mathrm{PO}_{4}(10 \mathrm{mM}) \text { :acetonitrila } \\
(3: 1)\end{array}$ & 1,0 & $100 \mu \mathrm{L}$ & ------- & ------ & ------- \\
\hline $\begin{array}{l}\text { MEHVAR, } \\
2000 \mathrm{~b}\end{array}$ & $\begin{array}{l}5 \mathrm{mg} / \mathrm{Kg} \\
\text { (MP eq.) }\end{array}$ & $\begin{array}{l}\text { Plasma } \\
\text { de rato }\end{array}$ & $\begin{array}{c}\text { MP e } \\
\text { DEX-MPS }\end{array}$ & 14,5 & Ac perclórico & $\begin{array}{l}\text { Coluna } \\
\text { em gel }\end{array}$ & Isocrático & 250 & $\begin{array}{c}\text { Água:acetonitrila:ác. acético } \\
\text { glacial }(75: 25: 0,2)\end{array}$ & 1,0 & $100 \mu \mathrm{L}$ & $\begin{array}{c}2 \\
\mu \mathrm{g} / \mathrm{mL}\end{array}$ & Trietilamina & 3 ratos \\
\hline $\begin{array}{l}\text { SHAH \& } \\
\text { WEBER, } 1985\end{array}$ & $\begin{array}{l}3,10,30 \\
\mathrm{mg} / \mathrm{Kg}(\mathrm{IR})\end{array}$ & $\begin{array}{l}\text { Plasma } \\
\text { de cão }\end{array}$ & $\begin{array}{c}\text { MP, sal } \\
\text { sódico de MP }\end{array}$ & -------- & $\begin{array}{l}\text { Ác. Acético } 25 \% \\
\text { aquoso }\end{array}$ & $\begin{array}{c}\mathrm{C}_{8} \\
\text { pré- } \\
\text { coluna } \\
\mathrm{C}_{8} \\
\end{array}$ & Isocrático & 243 & $\begin{array}{c}\text { Água:acetonitrila: } \\
\text { metanol:dimetil-octilamina:ác. } \\
\text { acético } \\
(65,5: 34: 0,4: 0,04: 0,04)\end{array}$ & 1,0 & $200 \mathrm{~L}$ & ------- & $\begin{array}{l}\text { Metil éster do } \\
\text { fenetilcarbamato }\end{array}$ & 1 cão \\
\hline $\begin{array}{l}\text { SHAH et al., } \\
1987\end{array}$ & $\begin{array}{l}10-3000 \\
\mathrm{mg}(\mathrm{IR})\end{array}$ & Plasma & $\begin{array}{c}\text { MP, sal } \\
\text { sódico de MP }\end{array}$ & ----- & Clorofórmio & $\begin{array}{c}\mathrm{C}_{18} 5 \mu \mathrm{m} \\
2504,5 \\
\mathrm{~mm} \mathrm{DI} \\
\text { pré- } \\
\text { coluna } \\
\mathrm{C}_{8}\end{array}$ & Isocrático & 243 & $\begin{array}{l}\text { Água:acetonitrila: } \\
\text { isopropanol }(71: 19,9: 10)\end{array}$ & 1,5 & $1,0 \mathrm{~mL}$ & ------- & $\begin{array}{l}\text { Propil-p- } \\
\text { hidroxibenzoato }\end{array}$ & 6 \\
\hline $\begin{array}{l}\text { VREE et al., } \\
1999\end{array}$ & $1 \mathrm{~g} / \mathrm{dia}$ & $\begin{array}{c}\text { Plasma e } \\
\text { urina }\end{array}$ & $\begin{array}{c}\text { MP e } 8 \\
\text { metabólitos }\end{array}$ & ינ-יב- & Ac perclórico & $\begin{array}{c}\mathrm{C}_{8} \mathrm{e} \\
\text { pré- } \\
\text { coluna } \\
\mathrm{C}_{8} \\
\end{array}$ & Gradiente & 248 & $\begin{array}{c}\text { Acetonitrila: } \\
\mathrm{KH}_{2} \mathrm{PO}_{4}(0,067 \mathrm{M}) \mathrm{pH} 4,5(3: 1) \\
\text { Variável na corrida }\end{array}$ & 1,5 & $150 \mathrm{~L}$ & $\begin{array}{c}0,1 \\
\mu \mathrm{g} / \mathrm{mL}\end{array}$ & ------ & $6 \mathrm{~F}$ \\
\hline
\end{tabular}




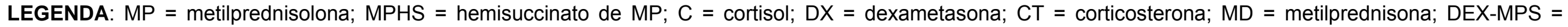

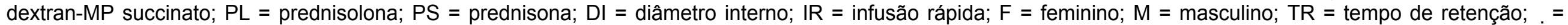
comprimento de onda.

Quadro 2. Parâmetros de validação da metilprednisolona e succinato de metilprednisolona encontrados na literatura internacional para amostras de plasma e soro sanguíneos, e comparados aos dados obtidos no presente estudo.

\begin{tabular}{|c|c|c|c|c|c|c|c|c|c|}
\hline Validação $\quad$ Referências & $\begin{array}{l}\text { AL-HABET } \\
\text { et al., } 1989\end{array}$ & KONG et al., 1988 & $\begin{array}{l}\text { LAWSON, } \\
1985\end{array}$ & $\begin{array}{l}\text { McWHINNEY } \\
\text { et al., } 1996\end{array}$ & $\begin{array}{l}\text { MEHVAR } \\
\text { et al., } 2000\end{array}$ & $\begin{array}{l}\text { SHAH et al., } \\
1985\end{array}$ & $\begin{array}{l}\text { SHAH et al., } \\
1987\end{array}$ & $\begin{array}{l}\text { VREE et al., } \\
1999\end{array}$ & RAMALHO, 2002 \\
\hline Concentração & ----- & 100,500 e $1000 \mathrm{ng} / \mathrm{mL}$ & $\begin{array}{c}13,4 ; 53,4 \\
133,5 \mathrm{~mol} / \mathrm{L}\end{array}$ & $\begin{array}{c}91-905 \\
\mathrm{ng} / \mathrm{mL}\end{array}$ & $\begin{array}{l}0,1 ; 0,25 ; 0,40 \\
1,0 ; 4,0 \quad \mathrm{~g} / \mathrm{mL}\end{array}$ & $\begin{array}{c}0,14-27,44 \\
\mathrm{~g} / \mathrm{mL}\end{array}$ & $\begin{array}{l}0,785 ; 3,14 \\
6,28 \mathrm{~g} / \mathrm{mL}\end{array}$ & $\begin{array}{l}1,263 ; 5,050 \\
20,29 \mathrm{~g} / \mathrm{mL}\end{array}$ & $\begin{array}{c}0,1 ; 0,25 ; 0,50 ; 1,0 ; 2,5 \\
5,0 ; 7,5 ; 10,0 \mathrm{~g} / \mathrm{mL}\end{array}$ \\
\hline Exatidão (\%) & ----- & ----- & ----- & ---- & ----- & $97,1-101,5$ & $98,4-103,1$ & $\begin{array}{c}\text { Intradia: } \\
3,4 ; 8,7 ; 0,3 \\
\text { Entre dias: } \\
7,6 ; 8,3 ; 0,4\end{array}$ & $\begin{array}{c}\text { MP: } 91-96,7 \\
\text { MPSS: } 91,7-97,3\end{array}$ \\
\hline $\begin{array}{l}\text { Precisão Intra-dia } \\
\text { (CV\%) }\end{array}$ & ----- & 5,9 a 2,8 & 7,$6 ; 5,6 ; 5,7$ & 5,$6 ; 4,1$ & $\begin{array}{c}5,3-3,6 \text { (MP) } \\
16-3,7 \text { (MPSS) }\end{array}$ & ----- & $<10$ & 6,$7 ; 3,0 ; 0,5$ & $\begin{array}{c}\text { MP: } 3,16-5,46 \\
\text { MPSS: } 3,55-6,65\end{array}$ \\
\hline $\begin{array}{l}\text { Precisão Interdia } \\
\text { (CV\%) }\end{array}$ & ----- & 6,5 e 2,3 & 15,$1 ; 8,2 ; 8,6$ & 9,$5 ; 5,0$ & $\begin{array}{c}6,6-3,6 \text { (MP) } \\
9,3-6,9 \text { (MPSS) }\end{array}$ & ----- & $<10$ & 2,$5 ; 2,9 ; 0,6$ & $\begin{array}{c}\text { MP: } 4,10-5,88 \\
\text { MPSS: } 4,12-6,45\end{array}$ \\
\hline $\begin{array}{l}\text { Linearidade } \\
\text { (r ou r2) }\end{array}$ & ----- & ----- & --- & ---- & $\begin{array}{c}\geq 0,99 \\
(0,1-4,0 \quad \mathrm{~g} / \mathrm{mL})\end{array}$ & $\begin{array}{c}\text { Até } 25 \mathrm{~g} / \\
\mathrm{mL}\end{array}$ & Até $200 \mathrm{~g} / \mathrm{mL}$ & $\begin{array}{c}0,9996 \\
(1,25-20 \\
\mathrm{g} / \mathrm{mL})\end{array}$ & $\geq \quad \begin{array}{c}0,999 \\
0,1-10 \mathrm{~g} / \mathrm{mL}\end{array}$ \\
\hline Seletividade & ----- & $\begin{array}{l}\text { Avaliada caso-a-caso, } \\
\text { dependendo da aplicação. }\end{array}$ & ---- & ----- & ----- & ----- & ----- & ----- & Seletivo (vide texto) \\
\hline Abrangência & ----- & Potencialmente & ----- & ----- & ----- & ---- & ----- & ----- & Abrangente \\
\hline Robustez & ----- & ----- & ----- & ----- & ----- & ----- & ----- & ----- & Robusta \\
\hline $\begin{array}{l}\text { Estabilidade dos } \\
\text { analitos }\end{array}$ & ----- & $\begin{array}{l}\text { Adequada em solventes } \\
\text { orgânicos }\end{array}$ & ----- & -.---. & ----- & ----- & ----- & ------ & Adequada: \\
\hline Recuperação (\%) & ----- & 61,5 & 62,$1 ; 63,9 ; 64,7$ & ----- & $\begin{array}{c}90 \text { (MP) } \\
83 \% \text { (MPSS) }\end{array}$ & $99 \pm 3$ & ----- & ----- & $\begin{array}{l}\text { MP: } 88 \pm 6 \% \\
\text { MPSS: } 85 \pm 5 \%\end{array}$ \\
\hline Resolução & ----- & $\geq 1,5$ & $\geq 1,5$ & Adequada & Adequada & Adequada & Adequada & ----- & $\geq 1,5$ \\
\hline $\begin{array}{l}\text { Limite de detecção } \\
\text { (LOD) } \\
\text { Limite de }\end{array}$ & ----- & ----- & ----- & ---- & ----- & ----- & $7 \mathrm{ng} / \mathrm{mL}$ & $0,10 \mathrm{~g} / \mathrm{mL}$ & $0,10 \mathrm{~g} / \mathrm{mL}$ \\
\hline quantificação (LOC) & ------ & ----- & --- & ---- & ----- & ----- & ----- & ----- & $0,25 \mathrm{~g} / \mathrm{mL}$ \\
\hline
\end{tabular}

Legenda: Concentração = intervalo de concentração do analito (MP ou MPSS), no qual foram obtidos os parâmetros de validação; C = concentração; MP = metilprednisolona; MPSS = succinato de metilprednisolona. 


\section{DISCUSSÃO}

Das 11 técnicas apresentadas no quadro 1, quatro (LAWSON, 1985; SHAH et al., 1987; SHAH \& WEBER, 1985; VREE et al., 1999) foram desenvolvidas para quantificar a MP em plasma de pacientes que receberam dose igual ou superior a $1000 \mathrm{mg}$, em esquema terapêutico semelhante ao deste estudo. Salienta-se que no estudo de SHAH \& WEBER (1985), o plasma utilizado foi de cão.

A maioria das técnicas apresentadas (LAWSON, 1985; KONG et al., 1988; McWHINNEY et al., 1996; MEHVAR, 2000b; VREE et al., 1999; HOPKINS et al., 1992) não quantifica o sal succinato sódico da MP, que foi também objetivo deste trabalho, de fundamental importância para estabelecer a relação cinética entre a MP e sua pró-droga para cada paciente.

O tempo de retenção (TR) da MP e da MPSS são referidos em um estudo (MEHVAR, 1999), enquanto que o tempo de retenção da MP pode ser observado em quatro outros artigos (LAWSON, 1985; McWHINNEY et al., 1996; MEHVAR et al., 2000; MEHVAR, 2000b). No caso destes três últimos, o tempo de retenção da MP é superior ao obtido na presente técnica analítica, chegando, no caso do estudo de McWHINNEY et al. (1996), a ser quase 5 vezes maior do que o TR aqui apresentado, o que torna onerosa e dispendiosa a análise de amostras de sangue em coleta seriada para estudos farmacocinéticos.

Os métodos de extração dos fármacos do material biológico são variados. Entretanto, pode-se verificar que os procedimentos de extração em fase líquida são mais freqüentes, sendo que o ácido perclórico, o diclorometano e o acetato de etila são utilizados em mais de um estudo (LAWSON, 1985; KONG et al., 1988; MEHVAR et al., 2000a; MEHVAR 2000b; VREE et al., 1999; AL-HABET \& ROGERS, 1989; HOPKINS et al., 1992). Escolheu-se o acetato de etila para extração dos corticosteróides desta técnica, por ser um solvente de baixo custo, fácil aquisição, volátil a baixas temperaturas, facilmente inativado (diminuindo risco de contaminação ambiental), bom precipitante de proteínas plasmáticas, prescindindo da necessidade de procedimentos de clean up demorados e custosos e não exige autorização especial para a sua aquisição no mercado brasileiro.

Dos 11 artigos citados, 7 utilizaram colunas cromatográficas em fase reversa (LAWSON, 1985; SHAH et al., 1987; SHAH \& WEBER, 1985; McWHINNEY et al., 1996; MEHVAR et al., 2000a; VREE et al., 1999; HOPKINS et al., 1992), existindo dois artigos utilizando coluna em fase normal (KONG et al., 1988; AL-HABET \& ROGERS, 1989) e dois utilizando coluna de exclusão molecular (MEHVAR, 2000b; MEHVAR, 1999). Nota-se, claramente, a preferência por colunas em fase reversa, possivelmente pela facilidade de aquisição e pela qualidade dos cromatogramas obtidos, notadamente pela resolução e seletividade das mesmas.

O comprimento de onda utilizado para leitura dos picos da MP variou entre os estudos (243 a $254 \mathrm{~nm}$ ), sendo o de $254 \mathrm{~nm}$ o mais freqüente, e também o utilizado neste trabalho, por representar o máximo de absortividade molar da metilprednisolona e boa absortividade do éster succinato de metilprednisolona e do padrão interno, a triancinolona (AL-HABET \& ROGERS, 1989).

Em cada um dos trabalhos supracitados desenvolveu-se fases móveis diferentes para a eluição dos analitos no material biológico, havendo preferência para modificadores de fase móvel de caráter ácido, o que favorece a resolução dos corticosteróides (KONG et al., 1988; SHAH \& WEBER, 1985; MEHVAR, 2000b; AL-HABET \& ROGERS, 1989; HOPKINS et al., 1992). Há 4 fases móveis que utilizam tampão salino (LAWSON, 1985; MEHVAR et al., 2000a; MEHVAR, 1999; VREE et al., 1999), passíveis de comprometer o sistema de bombeamento do cromatógrafo líquido, além de haver 2 artigos que apresentam fases móveis com 4 e 5 componentes na mistura (KONG et al., 1988; SHAH \& WEBER, 1985), o que aumenta as chances de erro e de variação nos resultados obtidos, além de favorecer ao aumento da pressão do sistema cromatográfico, contribuindo para a diminuição da vida útil do equipamento (QUATTROCCHI, 1992).

O fluxo utilizado para eluição da fase móvel oscilou de $1,0 \mathrm{~mL} / \mathrm{min}$ a $2,5 \mathrm{~mL} / \mathrm{min}$, contra apenas $0,6 \mathrm{~mL} / \mathrm{min}$ na técnica ora validada, o que contribui para o aumento da vida útil do equipamento cromatográfico, pela menor pressão no sistema cromatográfico (QUATTROCCHI, 1992).

A maioria das referências apresentadas não cita o limite de detecção do fármaco (KONG et al., 1988; SHAH et al., 1987; SHAH \& WEBER, 1985; McWHINNEY et al., 1996; MEHVAR, 1999; AL-HABET \& ROGERS, 1989). Entretanto, há valores que variam entre $2 \mathrm{ng} / \mathrm{mL}$ e $2 \mu \mathrm{g} / \mathrm{mL}$, dependendo do volume de amostra biológica utilizado, do loop no injetor do equipamento cromatográfico, do procedimento extrativo e de características relacionadas ao 
ganho do equipamento analítico. O LOD da presente técnica foi de $0,1 \mathrm{~g} / \mathrm{mL}$ e o $\mathrm{LOQ}$ de $0,25 \mathrm{~g} / \mathrm{mL}$, adequados para os propósitos de análise plasmática da MP e MPSS em pacientes sob pulsoterapia.

As técnicas analíticas revisadas não apresentaram informações acerca da seletividade, descrita neste trabalho e utilizada como parâmetro na validação bastante útil quando são realizadas análise em pacientes que, quase que invariavelmente, recebem outros medicamentos simultaneamente.

Em relação ao padrão interno utilizado nas técnicas apresentadas há uma variedade de substâncias do grupo dos corticosteróides, sendo que a triancinolona, utilizada nesta técnica, foi também utilizada nos estudos de MEHVAR et al. (2000a) e AL-HABET \& ROGERS (1989). No presente experimento, a triancinolona apresentou boa resposta no comprimento de onda escolhido, além de apresentar um baixo tempo de retenção e a não interferência com a metilprednisolona e seu éster succinato.

A maior parte dos artigos não apresentou resultados de pacientes humanos (SHAH \& WEBER, 1985; McWHINNEY et al., 1996; MEHVAR et al., 2000a; MEHVAR, 2000b; MEHVAR, 1999; HOPKINS et al., 1992). Nos estudos que envolveram humanos, o número de pacientes variou de 1 a 6 , mormente pela dificuldade em preencher os critérios de seleção.

Para facilitar e aprofundar a discussão sobre a validação da presente técnica analítica, apresentam-se no quadro 2 os valores encontrados na literatura internacional, bem como os da presente técnica. Nenhum dos autores citados no QUADRO 2 apresenta todos os parâmetros de validação analítica ora apresentados neste trabalho, sendo que as técnicas analíticas têm propósitos variados (desde quantificação de metilprednisolona sob baixas até altas doses) e algumas incluem dosagem de hormônios circulantes (cortisol, corticosterona). Entretanto, os autores que apresentaram parâmetros de validação tais como exatidão, precisão intradia e interdia, linearidade e recuperação (KONG et al., 1988; LAWSON, 1985; McWHINNEY et al., 1996; MEHVAR et al., 2000; SHAH et al., 1987; VREE et al., 1999), demonstraram que os valores obtidos estão dentro de limites aceitos internacionalmente (PENG, 1990; BRESSOLLE et al., 1996).

Valores numéricos para limite de detecção foram citados por apenas dois autores (SHAH et al., 1987; VREE et al., 1999) e o limite de quantificação das técnicas analíticas, bem como parâmetros de sensibilidade, não foram sequer citados. Os parâmetros seletividade, abrangência, robustez e estabilidade dos analitos foram apresentados parcimoniosamente ou negligenciados. Quanto ao parâmetro seletividade, há citação da necessidade de obtê-lo conforme aplicações das técnicas analíticas (KONG et al., 1988). De um modo geral, a maior preocupação dos autores foi com a repetibilidade (precisão intralaboratorial) e com a reprodutibilidade (precisão interlaboratorial) de suas técnicas analíticas, ressalvando as restrições das aplicações atribuídas às mesmas.

Quanto às aplicações desta técnica analítica, pode ser bastante útil na dosagem plasmática de MP e MPSS, além de outros corticosteróides (desde que validados), para fins de estudos cinéticos, de controle terapêutico ou em casos de intoxicação por tais agentes.

\section{CONCLUSÃO}

A técnica analítica apresentou parâmetros de validação condizentes com recomendações internacionais e com a legislação sanitária vigente no país. A técnica em foco apresentou excelente linearidade, além de boa precisão intradia e interdia, com coeficiente de variação bem menor que os $15 \%$ aceitáveis estatisticamente.

Assim, a presente metodologia analítica tem sustentação técnica para que possa ser utilizada na quantificação plasmática da metilprednisolona e do succinato sódico de metilprednisolona em pacientes sob pulsoterapia e, também, em outras situações que envolvam casos de intoxicação aguda e de controle terapêutico de medicamentos, além de possuir aplicações no desenvolvimento de estudos fármaco e toxicocinéticos do supracitado fármaco.

\section{REFERÊNCIAS BIBLIOGRÁFICAS}


AL-HABET, SM; ROGERS, HJ. Two chromatographic methods for the determination of corticosteroids in human biological fluids: pharmacokinetic applications. J. Pharm. Sci., v. 78, n. 8, p. 660-6, 1989a.

AL-HABET, SM; ROGERS, HJ. Methylprednisolone pharmacokinetics after intravenous and oral administration. Br. J. Clin. Pharmacol., v. 27, n. 3, p. 285-90, 1989b.

BERTONI, M; BRUGNOLO, F; BERTONI, E et al. Long term efficacy of righ-dose intraveous methylprednisolone pulses in activelupus nephrits. A 21-mouth prospective study. Scand. Jornal Rheumatology, 1994.

BRASIL. AGÊNCIA NACIONAL DE VIGILÂNCIA SANITÁRIA. Resolução n. ${ }^{\circ} 391$, de 9 de agosto de 1999. Aprova o Regulamento Técnico para Medicamentos Genéricos. In: Diário Oficial da União, Brasília, 19 nov. 1999.

BRASIL. AGÊNCIA NACIONAL DE VIGILÂNCIA SANITÁRIA. Manual de boas práticas em biodisponibilidade e bioequivalência, v. 1, p. 71, Brasília, 2002.

BRESSOLLE, F., BROMET-PETIT, M.; AUDRAN, M. Validation of liquid chromatographic and gas chromatographic methods: Application to pharmacokinetics. J. Chromatogr. B, v. 686, p.3-10, 1996.

BUDAVARI, S. et al. THE MERCK INDEX - Na Encyclopedia of Chemicals, Drugs and Biologicals, 11th Edition, Merck \& Co., Inc., Rahway, NJ, USA, 1989. p. 960.

CUNHA, LC. Disposição cinética dos estereoisômeros do R,S-sotalol em miocardiopatas crônicos portadores de taquiarritmias cardíacas. São Paulo: USP, 1998. Tese (Doutorado em Fármaco e medicamentos: produção e controle), Faculdade de Ciências Farmacêuticas, Universidade de São Paulo, 1998. p. 111.

HOPKINS, NK; WAGNER, CM; BRISSON, J; ADDISON, TE. Validation of the simultaneous determination of methylprednisolone and methylprednisolone acetate in human plasma by high-performance liquid chromatography. J. Chromatogr. B, v. 577, p. 87-93, 1992.

ICHIKAWA, Y; HASHIMOTO, H; KASHIWAZAKI, S et al. Methylprednisolone pulse therapy for SLE patients with CNS disorder. Ryumachi, v. 34, n. 4, p. 733-43, 1994.

ITO, S; KUSUNOKI, Y; OKA, T; ITO, Y et al. Pharmacokinetics of higt-dose methylprednisolone in children. Dev. Pharmacol. Ther., v. 19, n. 2-3, p. 99-105, 1992.

KONG, AH-NG; SLAUGHTER, RL; JUSKO, WJ. Simultaneous analysis of methylprednisolone hemisuccinate, cortisol and methylprednisolone by normal-phase high-performance liquid chromatography in human plasma. $\mathrm{J}$. Chromatogr. B, v. 432, p. 308-314, 1988.

LAWSON, GJ. Rapid method for the measurement of methylprednisolone ans its hemisuccinate in plasma and urine following "pulse therapy" by high-performance liquid chromatography. J. Chromatogr. B, v. 342, p. 251-260, 1985.

LILLICH, JD; BERTONE, AL; SCHMALL, LM; RUGGLES, AJ et al. Plasma, urine and synovialfluid disposition of methylprednisolone acetate and isoflupredone acetate after intra-articular administration in horses. Am. J. Vet. Res., 1996.

McWHINNEY, BC; WARD, G; HICKMAN, PE. Improved HPLC method for simultaneous analysis of cortisol, 11deoxicortisol, prednisolone, methylprednisolone, and dexamethasone in serum and urine. Clin. Chem., v. 42, n. 6, parte 1, p. 979-81, 1996.

MEHVAR, R. High-performance size-exclusion chromatographic analysis of dextran-methylprednisolone hemisuccinate in rat plasma. J. Chromatogr. B, v. 744, p. 293-298, $2000 \mathrm{~b}$.

MEHVAR, R. Simultaneous analysis of dextran-methylprednisolone succinate, methylprednisolone succinate and methylprednisolone by size-exclusion chromatography. Journal of Pharmaceutical and Biomedical Analysis. v. 19, p. 785-792, 1999.

MEHVAR, R; DANN, RO; HOGANSON, DA. Simultaneous analysis of methylprednisolone succinate, and endogenous corticosterone in rat plasma. Journal of Pharmaceutical and Biomedical Analysis. v. 22, p. 1015-1022, 2000a.

MURPHY, EJ; SLIVKA, AP; ROSENBERGER, TA et al. Higt-performance liquid chromatography separation and quantification of methylprednisolone from rat brain. Anal. Biochem., v. 209, n. 2, 339-42, 1993.

PENG, GW; CHIOU, WL. Analysis of drugs and other toxic substances in biological samples for pharmacokinetic studies. J. Chromatogr., Amsterdam, v. 531, p. 3-50, 1990.

QIN, Y; LIANG, D; ZENG, J; MAO, W. Determination of hydrocortisone and methylprednisolone in plasma by reversed-phase HPLC. Hua-Hsi-I-Ko-Ta-Hsueh-Hsueh-Pao, v. 22, n. 3, p. 270-3, 1991. 
QUATTROCCHI, O. A., ANDRIZZI, S. A., LABA, R. F. Introducción a la HPLC - aplicación y práctica. Buenos Aires: Merck, 1992. p. 422

RAMALHO, PIS. Farmacocinética clínica da metilprednisolona sob pulsoterapia em pacientes com lúpus eritematoso sistêmico. Goiânia: UFG, 2002. Dissertação (Mestrado em Biologia, área de Fisiologia, sub-área de Fisiologia Animal), Instituto de Ciências Biológicas, Universidade Federal de Goiás, 2002.

RÊGO, J; OLIVEIRA, FM; VILELA, RG et al. Pulsoterapia no lúpus eritematoso sistêmico: resultados em quarenta pacientes. Rev. Bras. de Reumatol., v. 34, n. 6, p. 298-302, 1994.

SHAH, JA; WEBER, DJ. High-performance liquid chromatographic assay for methylprednisolone and its soluble produg esters in dog plasma. J. Chromatogr. B, v. 344, p. 41-49, 1985.

SHAH, JA; WEBER, DJ; BOTHWELL, BE. High-performance liquid chromatography determination of methylprednisolone and methylprednisolone $21-$ [8 - [methyl - (2 - sulfoethyl) amino] - 8 - oxoctanoate] sodium salt in human plasma. J. Chromatogr. B, v. 414, p. 1-10, 1987.

VREE, TB; LAGERWERF, AJ; WISSEN, C; JONGEN, PJ. High-performance liquid chromatographic analysis, preliminary pharmacokinetics, metabolism and renal excretion of methylprednisolone with its C6 and C20 hydroxy metabolites in multiple sclerosis patients receiving high-dose pulse therapy. J. Chromatogr. B, v. 732, p. 337-348, 1999. 